\title{
COMPARATIVE STUDY OF CLINICAL EFFECT OF CUFFED VS UNCUFFED ENDOTRACHEAL TUBE IN NEONATES UNDERGOING SURGERY UNDER GENERAL ANAESTHESIA
}

\author{
Priti Banerjee1, Dipasri Bhattacharya², Soma Mukhopadhyay33, Mohanchandra Mandal ${ }^{4}$ \\ 1Postgraduate Trainee, Department of Anaesthesiology and Critical Care, R. G. Kar Medical College and Hospital, Kolkata, West Bengal, \\ India. \\ 2Professor and HOD, Department of Anaesthesiology and Critical Care, R. G. Kar Medical College and Hospital, Kolkata, West Bengal, \\ India. \\ ${ }^{3}$ Associate Professor, Department of Anaesthesiology and Critical Care, R. G. Kar Medical College and Hospital, Kolkata, West Bengal, \\ India. \\ ${ }^{4}$ Associate Professor, Department of Anaesthesiology and Critical Care, N. R. S. Medical College and Hospital, Kolkata, West Bengal, \\ India.
}

ABSTRACT

\section{BACKGROUND}

The use of cuffed Endotracheal Tube (ET tube) in neonates is not much popular, because of the fear of airway complications. But this traditional thinking is now changing with cuffed tube becoming more acceptable and popular.

This study was conducted to compare postoperative airway complications and tube exchange rate in neonates intubated with cuffed or uncuffed endotracheal tube.

\section{MATERIALS AND METHODS}

Fifty neonates who were undergoing surgery under general anaesthesia with endotracheal intubation were included in this randomised active-controlled single-blinded study. They were randomly allocated into two groups to receive intubation with either cuffed ET tube (Group C, $n=25$ ) or uncuffed ET tube (Group U, n= 25). Data from 23 neonates in each group was available for analysis with the relaxation of study protocol. The tube exchange rate and incidences of postoperative airway adverse events such as hoarse cry, laryngospasm and apnoeic spell were noted in both the groups.

\section{RESULTS}

Mean ages of neonates in both groups were comparable (13.5 days and 12.7 days, respectively). The tube exchange rate was considerably less in cuffed tube group compared with uncuffed tube group (4.3\% versus 65\%, respectively, $p=0.0000002$ ). Incidence of postoperative airway adverse events was also found to be significantly less in cuffed tube group compared with uncuffed group (4\% versus $43 \%$, respectively, $\mathrm{p}=0.00009$ ).

\section{CONCLUSION}

The use of cuffed ET tube in neonates reduces the need for tube exchange and reduces postoperative adverse events, such as hoarse cry and apnoeic spell compared with the use of uncuffed ET tube.

\section{KEY WORDS}

Neonate, Endotracheal Tube, Intubation, Morbidity, Postoperative Period.

HOW TO CITE THIS ARTICLE: Banerjee P, Bhattacharya D, Mukhopadhyay S, et al. Comparative study of clinical effect of cuffed vs uncuffed endotracheal tube in neonates undergoing surgery under general anaesthesia. J. Evolution Med. Dent. Sci. 2018;7(35): 3873-3877, DOI: $10.14260 /$ jemds/2018/867

\section{BACKGROUND}

Majority of neonatal and infant surgical procedures are being carried out under general anaesthesia with endotracheal intubation to provide safety and to allow adequate ventilation. Two articles were published as early as in 1965 in the British Journal of Anaesthesia regarding the use of endotracheal intubation in paediatric patients for prolonged ventilation. ${ }^{[1,2]}$ It reported about the incidence of severe subglottic narrowing in a 2 -year-old child ventilated for prolonged period.

'Financial or Other Competing Interest': None.

Submission 16-07-2018, Peer Review 10-08-2018,

Acceptance 16-08-2018, Published 27-08-2018.

Corresponding Author:

Mohanchandra Mandal,

DKB Green, Block-A, Flat-2H, 57/4,

B. T. Road, Kolkata-700002,

West Bengal, India.

E-mail: drmcmandal@gmail.com

DOI: $10.14260 /$ jemds $/ 2018 / 867$

(c) $($ ) $($ ) $\Theta$
In the very next year (1966), in a seminal article in British Medical Journal, Stocks JG[3] pointed out the importance of selecting appropriate size of tracheal tube as fundamental to the success of prolonged nasal intubation in children. Later, Battersby et al[4] concluded that there was no subglottic stenosis from proper use of endotracheal tubes (ET tube) for long-term intubation. Black et al[5] confirmed this in a study as early as in 1990 . Hence, it is very important to choose proper sized ET tube in children requiring mechanical ventilation to reduce post-intubation adverse events.

The loose areolar tissue covering the anterior surface of epiglottis and cricoid is very prone to develop oedema. Any compression to this mucosa by an oversized ET tube may cause mucosal oedema, subsequently fibrosis leading to stenosis. Few of the studies also demonstrated that an oversized ET tube use was associated with development of ductal cysts and scars bridging the vocal cords.[6] Therefore, uncuffed ET tubes have been used for many years to minimise tracheal and laryngeal injury. However uncuffed tubes have many additional disadvantages, i.e. requirement of multiple 
changes to make a complete seal to avoid air leak.[7] Chance of displacement is also high. Use of throat pack is related to hoarse cry and delayed oral feeding. In addition to this, monitoring of ventilator parameters and exhaled volumes become difficult.[8] Therefore, a great debate has developed while choosing between cuffed and uncuffed ET tube.

Cuffed tube has not been very popular because of the fear that the cuff would cause airway mucosal injury, leading to subglottic stenosis.[9-11] Contrary to the previous practice, UK practice has changed now with cuffed tube becoming more acceptable for use in children. ${ }^{[7]}$ Although, the cuffed ET tube has several advantages, the necessity of costly microcuff, frequent cuff pressure monitoring etc., remain to be prerequisite for ensuring its benefits. Hence, the efficacy of cuffed ET tube in neonatal setting still remains to be a controversial issue. Therefore, this study was conducted to compare the tube exchange rate (Primary outcome), incidence of post-extubation morbidities (Secondary outcome) in neonates, undergoing surgery under general anaesthesia with tracheal intubation with either cuffed or uncuffed ET tube.

\section{MATERIALS AND METHODS}

The present randomised active-controlled single-blinded study was designed to compare the ET tube exchange rate between neonates undergoing surgery under general anaesthesia using either cuffed ET tube or uncuffed ET tube. Weiss et $\mathrm{al}^{8}$ observed that tube exchange rate was $30.8 \%$ with uncuffed tubes, whereas it was only $2.1 \%$ using cuffed tubes. Consultation with other literature reveals that the reported incidence of tube exchange rate varies from $25 \%{ }^{[12]}$ to $37.6 \%{ }^{[13]}$ in the uncuffed tubes and $1.97 \%{ }^{[14]}$ to $2.1 \%{ }^{[8]}$ in the cuffed group. Setting the power of study at $80 \%$, Confidence Interval (CI) at $95 \%$ and allowing type 1 error of $5 \%$, the estimated sample size was found to be 22.7 for each group using the following formula $\left(n=2(Z \alpha+Z \beta)^{2} \times\left[p_{1}\left(1-p_{1}\right)\right.\right.$ $\left.+p_{2}\left(1-p_{2}\right)\right) /\left(p_{1}-p_{2}\right)^{2]}$. Considering the possible dropout, we recruited 25 neonates for each group.

Neonates who underwent surgery under general anaesthesia with American Society of Anesthesiologists (ASA) physical status I-II were included in the present study. Both male and female neonates were included. Neonates conforming to ASA physical status more than II with known airway anomalies, known or suspected difficult intubation were excluded from the study. Neonates, who required postoperative ventilation in ICU were also excluded from this study.

The study was initiated after obtaining approval from Institute's Ethics Committee. The study protocol was explained to the legally acceptable guardians to obtain their consent. After getting written consent from guardians, neonates were randomly allocated into two groups for intubation with either cuffed ET tube (Group C, n= 25) or uncuffed ET tube (Group $\mathrm{U}, \mathrm{n}=25$ ) according to the computer-generated codes (Random numbers). Allocation concealment was achieved by placing the randomisation sequence for each technique (Random numbers) in sequentially numbered sealed opaque envelopes. Each time before the start of anaesthesia, one envelope is opened to get the random number indicating the group.

Standard protocol of induction of general anaesthesia in neonates was followed with inhalation of $6-8 \%$ sevoflurane in $100 \%$ oxygen. Intravenous access was secured. Precordial stethoscope was used in every case to monitor the ventilation and heart rate. Monitoring of vital parameters such as systolic and diastolic blood pressure (SBP, DBP), heart rate, oxygen saturation, ECG, capnogram and core temperature was performed.

After induction of hypnosis, face mask ventilation was confirmed and thereafter muscle paralysis was achieved with injection atracurium $(0.5 \mathrm{mg} / \mathrm{kg})$. Then tracheal intubation was performed under direct laryngoscopy by oral route. All intubations were done by expert paediatric anaesthesiologist.

For premature neonates cuffed ET tubes with internal diameter of $2.5 \mathrm{~mm}$ and for mature neonates (more than 2.5 $\mathrm{kg}$ body weight), cuffed ET tubes with internal diameter of 3 $\mathrm{mm}$ were used. Then the cuff was inflated with the use of cuff pressure manometer (Portex). Cuff pressure was limited to $15 \mathrm{mmHg}$ with the help of pressure release valve. During steady state ventilation, the cuff pressure was gradually reduced until there was an audible leak at the mouth of the neonate. Then the cuff pressure was increased till the disappearance of that leak. The cuff pressure at this moment is called the 'minimal sealing pressure,' which was maintained. This minimal cuff pressure which was required to seal the airway was recorded. Throat packs were given to prevent air leak when uncuffed ET tubes were selected as per study protocol. Lungs were checked for bilateral equal breath sounds and position of the ET tube was confirmed by bilateral equal breath sounds and capnography. Once it is confirmed that a proper sized ET tube has been used in either groups, adequacy of sealing was tested by mechanical ventilation. The tube was secured with adhesive tape. General anaesthesia was maintained according to standard protocol using $\mathrm{N}_{2} \mathrm{O}: \mathrm{O}_{2}=2: 1$, sevoflurane $(1-2 \%)$, injection atracurium.

An intraoperative analgesia was provided with injection fentanyl (2 microgram/ kg), paracetamol suppository (15 $\mathrm{mg} / \mathrm{kg}$ ). During intraoperative period, all the vital parameters were assessed. Any need for tube exchange was recorded (Primary outcome).

On completion of surgery, extubation was done after giving antagonist of residual neuromuscular block (Injection neostigmine- $0.05 \mathrm{mg} / \mathrm{kg}$ and Injection glycopyrrolate$0.01 \mathrm{mg} / \mathrm{kg}$ ). Supplementary oxygen was provided in the postoperative period. All neonates were sent to neonatal care unit after extubation. The incidence of hoarse cry, laryngospasm, apnoeic spell, number of episodes of vomiting, time for first oral intake and any other complications in first 24 hours were recorded by an anaesthesiologist who was blinded to group allocation. Incidences of these morbidities were calculated for each group.

Tube exchange rate was taken as primary outcome and incidence of postoperative morbidities such as hoarse cry, laryngospasm and apnoeic spell were considered as secondary outcome measures. In the present study 'cuffed' ET tube (Newer method or Test) has been compared with 'uncuffed' ET tube (Control). As uncuffed ET tube is also a mode of therapy, it is acting as active control. Only the patients (Neonates) were unaware of therapy they received. Owing to the specific design of cuffed or uncuffed ET tube, it was not possible to make the primary investigator blind.

Data were charted on the Excel Workbook (Microsoft Office Home and Student 2007, Microsoft Corporation, One 
Microsoft Way in Redmond, WA 98052, USA) and analysed using Statistical Package for the Social Sciences (SPSS) for Windows (version 12.0, SPSS Inc., Chicago, IL, USA). Independent sample t-test (Unpaired t-test) was used to analyse numerical data such as age, weight, duration of surgery and duration of anaesthesia. Fisher's exact test was utilised to analyse categorical data such as tube exchange rate (number of tube exchange required) and incidence of postoperative morbidity such as hoarse cry, apnoeic spell, etc. All statistical tests were two-sided and were performed at a significance level of $p=0.05$. Hence, a $p<0.05$ was taken to be of statistical significance.

\section{RESULTS}

The study spanned over approximately eight months (From May 2017 to December 2017). Two neonates in cuffed tube group developed laryngospasm immediately after induction with sevoflurane. This stormy induction was managed accordingly with relaxation of study protocol. In the uncuffed group, one neonate had upper respiratory tract infection in the morning of operation day and another was excluded for having diarrhoea on the day of surgery. Data from those four neonates were not taken into consideration for analysis as there was violation of the study protocol. Hence, data from 23 neonates from each group were available for analysis. The two groups were comparable in respect to demographic data such as age, weight as well as duration of surgery and duration of anaesthesia [Table 1]. There was no significant difference in type of surgery performed in both the groups [Figure 1].

The primary outcome of the study, i.e. the tube exchange rate was higher with the use of uncuffed ET tube $(65 \%)$ in comparison to the use of cuffed ET tube (4.3\%). The difference was statistically significant $(p<0.05)$ [Table 2]. The incidence of postoperative hoarse cry was 39\% ( 9 out of 23) in neonates intubated with uncuffed ET tube compared with no such occurrence with the use of cuffed ET tube. It was also observed that one neonate of each group showed apnoeic spell. Though apnoeic spell was taken as a postoperative respiratory complication, it cannot be concluded that this complication was directly related to the type of tube (cuffed or uncuffed) used for intubation. But the overall incidence of postoperative morbidity was significantly higher in uncuffed tube (43\%) in comparison to the cuffed tube (4\%) [Table 3].

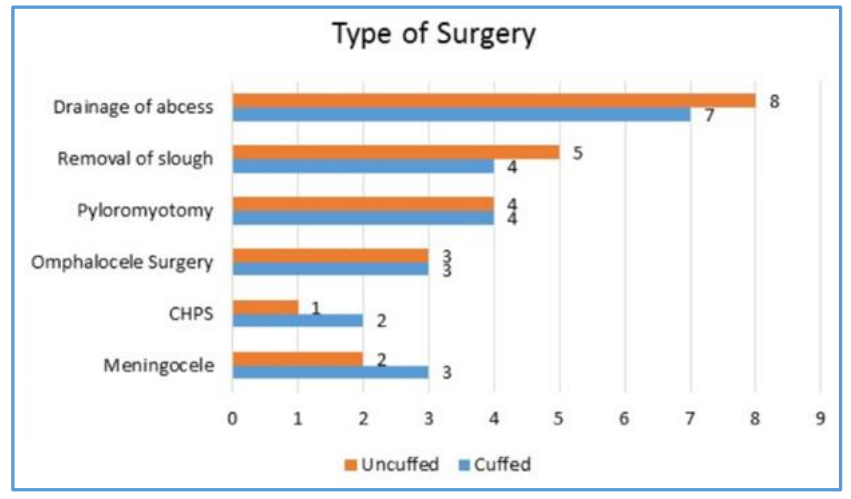

Figure 1. Type of Surgery

\begin{tabular}{|c|c|c|l|}
\hline Parameters & $\begin{array}{c}\text { Uncuffed } \\
(\mathbf{n = 2 3 )}\end{array}$ & $\begin{array}{c}\text { Cuffed } \\
(\mathbf{n = 2 3 )}\end{array}$ & P-value \\
\hline Age (days) & $\begin{array}{c}12.7 \pm 4.05 \\
(0.84)\end{array}$ & $\begin{array}{c}13.5 \pm 4.01 \\
(0.84)\end{array}$ & 0.472 \\
\hline Weight (kg) & $\begin{array}{c}2.71 \pm 0.23 \\
(0.047)\end{array}$ & $\begin{array}{c}2.66 \pm 0.22 \\
(0.046)\end{array}$ & 0.151 \\
\hline Duration of & $\begin{array}{c}36.08 \pm 12.96 \\
(2.7)\end{array}$ & $\begin{array}{c}32.95 \pm 14.44 \\
(3.01)\end{array}$ & 0.972 \\
\hline Surgery (min) & $\begin{array}{c}46.95 \pm 12.5 \\
(2.6)\end{array}$ & $\begin{array}{c}42.39 \pm 14.5 \\
(3.03)\end{array}$ & 0.77 \\
\hline Duration of & \begin{tabular}{c}
46.93 \\
\hline
\end{tabular}
\end{tabular}

Data is expressed as Mean \pm Standard Deviation (Standard Error of Mean). Unpaired t-test is used to compare the data. $P$ less than 0.05 is considered to be significant. Table 1. Demographic Data

\begin{tabular}{|c|c|c|c|c|c|}
\hline 总 & 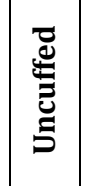 & 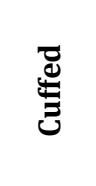 & 莺 & $\frac{0}{ٍ \pi}$ & 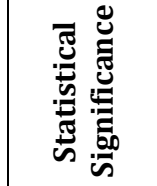 \\
\hline Once & 10 & 1 & \multirow{4}{*}{$\begin{array}{c}\text { Fishers' } \\
\text { Exact Test }\end{array}$} & \multirow{4}{*}{0.0000002} & \multirow{4}{*}{$\begin{array}{c}\text { Highly } \\
\text { Significant }\end{array}$} \\
\hline Twice & 5 & 0 & & & \\
\hline Total & 15 & 1 & & & \\
\hline $\begin{array}{l}\text { Percentage } \\
\text { of } \\
\text { Occurrence }\end{array}$ & $65 \%$ & $4.3 \%$ & & & \\
\hline
\end{tabular}

\begin{tabular}{|c|c|c|c|c|c|}
\hline 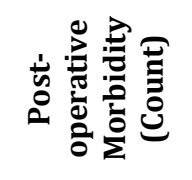 & 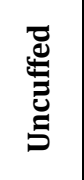 & 弟 & 莺 & $\begin{array}{l}\frac{0}{3} \\
\frac{\pi}{2} \\
2\end{array}$ & 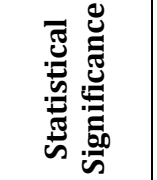 \\
\hline Hoarse Cry & 9 & 0 & \multirow{4}{*}{$\begin{array}{c}\text { Fishers' } \\
\text { Exact Test }\end{array}$} & \multirow{4}{*}{0.00009} & \multirow{4}{*}{$\begin{array}{c}\text { Highly } \\
\text { Significant }\end{array}$} \\
\hline Apnoeic Spell & 1 & 1 & & & \\
\hline Total & 10 & 1 & & & \\
\hline $\begin{array}{c}\text { Percentage of } \\
\text { Occurrence }\end{array}$ & $43 \%$ & $4 \%$ & & & \\
\hline
\end{tabular}

\section{DISCUSSION}

In the present study, the ET tube exchange rate was found to be $4.3 \%$ in cuffed tube group and $65 \%$ in the group receiving uncuffed ET tube. Khine et al[7] also found a lower tube exchange rate in groups receiving cuffed ET tube compared with uncuffed ET tube (1.2\% versus $23 \%$, respectively). Khine et al[7] also found that rate of reintubation required was $18 \%$ in cuffed tube versus $30 \%$ in uncuffed tube in children younger than 2 years. Similar type of observation was reported by Weiss et al,[8] where tube exchange rate was $2.1 \%$ in cuffed tube and $30.8 \%$ in uncuffed tube. Thus, it appears that the result of this study is consistent with the findings of the other previously reported studies. To prevent air leak in uncuffed tube, many a times throat pack is used to seal the leak. This technique results in a high incidence of delayed oral feeding and postoperative hoarse cry. The use of cuffed tubes reduces such problems as no additional throat pack is required to seal the airway.

In paediatric population, most of the surgeries are being carried out under general anaesthesia with endotracheal intubation. Previously uncuffed tubes were considered safe and were recommended in paediatric patients. But considering the problems associated with uncuffed ET tube, 
cuffed ET tube is now becoming acceptable also in the paediatric population. A few of the newer study revealed that in paediatric group, cricoid lumen is not at all circular, but elliptical.[15] When a round uncuffed ET tube is used there is considerable pressure over posterolateral wall of cricoids known as 'cricoid sealing.' If a proper size cuffed tube is used, the airway is sealed at the trachea known as 'tracheal sealing.'[16] There is limited study comparing 'cricoid sealing' with the 'tracheal sealing.'

Tracheal capillary mucosal perfusion pressure in adult age group is in the range of $25-30 \mathrm{mmHg}$. If the cuff pressure is maintained at $25 \mathrm{mmHg}$ or less, it ensures adequate seal to prevent aspiration as well as maintains mucosal perfusion. But in case of neonates and infants, this cuff pressure should be kept less than $20 \mathrm{mmHg}$ to prevent mucosal damage.[17-19] Hence, in the present study, the cuff pressure of ET tube was limited to $15 \mathrm{mmHg}$ using a cuff pressure manometer.

One of the most important considerations for an anaesthesiologist is to choose appropriately sized endotracheal tube that provides controlled ventilation without considerable airway injury. As the trachea of neonate is very short in length, a little movement of the uncuffed tube inside the trachea may cause dislodgement and repositioning of the ET tube leading to high tube exchange rate. Many a times, uncuffed ET tube may not fit properly causing air leak around the tube. An excessive air leak around the tube may lead to unreliable monitoring of ventilator parameters, exhaled volumes, end expiratory gases which may be utmost important in the critically ill neonates or child requiring ventilatory support.[20] The risk of aspiration is also high in case of excessive leak.[21,22]

In the present study, it was found that higher incidence of postoperative hoarse cry in neonates intubated with uncuffed ET tube (39\%) compared with no such occurrence with the use of cuffed ET tube. However, one neonate in both the groups showed apnoeic spell. So the incidence of postoperative morbidity was considerably higher in uncuffed ET tube group compared with cuffed ET tube group (43\% vs $4 \%$, respectively). This observation is in line with that of the previously stated studies. ${ }^{7,8}$ In the recent past, several studies have compared the effect of using cuffed and uncuffed tubes in children regarding the incidence of postoperative airway complications.[23-27] Holzki et al[10] reported that the incidence of laryngeal injury is higher in case of cuffed ET tube and they also stated that oversized cuffed ET tube was responsible for airway trauma. However, no study has demonstrated that proper sized cuffed ET tube has caused airway injury. Khine et al[7] did not find any increased incidence of croup in children intubated with cuffed ET tube. Newth et al[23] demonstrated no significance incidence of airway complications in cuffed ET tube. Risk of airway damage in cuffed tube is associated with oversized outer tube diameters, poorly designed cuffs, wrong placement of the tube and over-inflation of the cuff resulting in postoperative stridor. But proper size of the cuff, appropriate cuff inflation pressure and careful length adjustment may combat any postoperative airway morbidity. Furthermore uncuffed tubes may cause postoperative airway complication like hoarse cry, laryngospasm as throat pack is often used with uncuffed ET tube. In 2001, Murat et al[24] and James et al[25] reported no incidence of any postoperative airway complication among 15,000 paediatric patients intubated with cuffed ET tube.
Most of the previous studies showed significant results in favour of cuffed ET tube. A recent Cochrane review[27] analysed three trials comparing cuffed with uncuffed ET tubes. It found no difference in post-extubation stridor between the two groups in two trials involving 2734 children. Another trial involving 70 children revealed that the use of cuffed ET tube reduced the amount of anaesthetic gas consumption owing to overall less gas flow. However, the review pointed out that caution to be exercised while interpreting studies comparing the use of cuffed and uncuffed ET tubes owing to the variability in the designs among several studies. Evidence is accumulating regarding the use of newly designed, sophisticated microcuff ET tube.[28,29] Large, well-designed trials involving different geographical areas is warranted to clarify factors such as the ability of these cuffed ET tubes to curtail anaesthetic cost, respiratory adverse events, etc.

\section{CONCLUSION}

A proper sized cuffed ET tube provides better laryngeal seal with less tube exchange rate and also favourable adverse event profile compared with uncuffed ET tube.

\section{REFERENCES}

[1] Mcdonald IH, Stocks JG. Prolonged nasotracheal intubation. A review of its development in a paediatric hospital. Br J Anaesth 1965;37:161-73.

[2] Allen TH, Steven IM. Prolonged endotracheal intubation in infants and children. Br J Anaesth 1965;37(8):566-73.

[3] Stocks JG. Prolonged intubation and subglottic stenosis. Br Med J 1966;2(5523):1199-200.

[4] Battersby EF, Hatch DJ, Towey RM. The effects of prolonged naso-endotracheal intubation in children. A study in infants and young children after cardiopulmonary bypass. Anaesthesia 1977;32(2):154-7.

[5] Black AE, Hatch DJ, Nauth-Misir N. Complications of nasotracheal intubation in neonates, infants and children: a review of 4 years' experience in a children's hospital. Br J Anaesth 1990;65(4):461-7.

[6] Bauman NM, Benjamin B. Subglottic ductal cysts in the preterm infant: association with laryngeal intubation trauma. Ann Otol Rhinol Laryngeal 1995;104(12):9638.

[7] Khine HH, Corddry DH, Kettrick RG, et al. Comparison of cuffed and uncuffed endotracheal tubes in young children during general anesthesia. Anesthesiology 1997;86(3):627-31.

[8] Weiss M, Dullenkopf A, Fischer JE, et al. Prospective randomized controlled multi-centre trial of cuffed or uncuffed endotracheal tubes in small children. $\mathrm{Br} \mathrm{J}$ Anaesth 2009;103(6):867-73.

[9] Uejima T. Cuffed endotracheal tubes in pediatric patients. Anesth Analg 1989;68(3):423.

[10] Holzki J. Laryngeal damage from tracheal intubation. Paediatr Anaesth 1997;7(6):435-7.

[11] Eschertzhuber S, Salgo B, Schmitz A, et al. Cuffed endotracheal tubes in children reduce sevoflurance and medical gas consumption and related costs. Acta Anaesthesiol Scand 2010;54(7):855-8. 
[12] Hernández-Cortez E, Martínez-Bernal GF. Airway in the newborn patient. J Anesth Crit Care Open Access 2016;5(1):00172.

[13] Dorsey DP, Bowman SM, Klein MB, et al. Perioperative use of cuffed endotracheal tubes is advantageous in young pediatric burn patients. Burns 2010;36(6):85660.

[14] Shi F, Xiao Y, Xiong W, et al. Cuffed versus uncuffed endotracheal tubes in children: a meta-analysis. J Anesth 2016;30(1):3-11.

[15] Litman RS, Weissend EE, Shibata D, et al. Developmental changes of laryngeal dimensions in unparalyzed, sedated children. Anesthesiology 2003;98(1):41-5.

[16] Weiss M, Knirsch W, Kretschmar 0, et al. Tracheal tube-tip displacement in children during head-neck movement - a radiological assessment. Br J Anaesth 2006;96(4):486-91.

[17] Dullenkopf A, Gerber AC, Weiss M. Fit and seal characteristics of a new paediatric tracheal tube with high volume-low pressure polyurethane cuff. Acta Anaesthesiol Scand 2005;49(2):232-7.

[18] Weiss M, Gerber AC. Cuffed tracheal tubes in children things have changed. Pediatr Anesth 2006;16(10):1005-7.

[19] Weber T, Salvi N, Orliaguet G. Cuffed vs non-cuffed endotracheal tubes for pediatric anesthesia. Pediatr Anesth 2009;19(Suppl 1):46-54.

[20] Main E, Castle R, Stocks J, et al. The influence of tracheal tube leak on the assessment of respiratory function in ventilated children. Intensive Care Med 2001;27(11):1788-97.
[21] Gopalareddy V, He Z, Soundar S, et al. Assessment of the prevalence of microaspiration by gastric pepsin in the airway of ventilated children. Acta Paediatr 2008; 97(1):55-60.

[22] Browning DH, Graves SA. Incidence of aspiration with endotracheal tubes in children. J Pediatr 1983;102(4):582-4.

[23] Newth CJ, Rachmann B, Patel N, et al. The use of cuffed versus uncuffed endotracheal tubes in pediatric intensive care. J Pediatr 2004;144(3):333-7.

[24] Murat I. Cuffed tubes in children: a 3-year experience in a single institution. Paediatr Anaesth 2001;11(6):748-9.

[25] James I. Cuffed tubes in children. Paediatr Anaesth 2006;11:259-63.

[26] Mukhopadhyay S, Mukhopadhyay S, Bhattacharya D, et al. Clinical performance of cuffed versus uncuffed preformed endotracheal tube in pediatric patients undergoing cleft palate surgery. Saudi J Anaesth 2016;10(2):202-7.

[27] De Orange FA, Andrade RG, Lemos A, et al. Cuffed versus uncuffed endotracheal tubes for general anaesthesia in children aged eight years and under. Cochrane Database of Systematic Reviews 2017;11:CD011954.

[28] Mhamane R, Dave N, Garasia M. Use of Microcuff $₫$ endotracheal tubes in paediatric laparoscopic surgeries. Indian J Anaesth 2015;59(2):85-8.

[29] Bhardwaj N. Pediatric cuffed endotracheal tubes. J Anaesthesiol Clin Pharmacol 2013;29(1):13-18. 